\title{
Emergent Universe Scenario in the Einstein-Gauss-Bonnet Gravity with Dilaton
}

\author{
B. C. Paul *and S. Ghose ${ }^{\dagger}$ \\ Physics Department, North Bengal University \\ Dist : Darjeeling, PIN : 734 430, India.
}

\begin{abstract}
We obtain cosmological solutions which admit emergent universe (EU) scenario in the framework of Einstein Gauss-Bonnet gravity coupled with a dilaton field in four dimensions. The coupling parameter of the Gauss-Bonnet terms and the dilaton in the theory are determined for obtaining an EU scenario. The corresponding dilaton potential which admits such scenario is determined. It is found that the GaussBonnet (GB) terms coupled with a dilaton field plays an important role in describing the dynamics of the evolution of the early as well as the late universe. We note an interesting case where the GB term dominates initially in the asymptotic past regime, subsequently it decreases and thereafter its contribution in determining the dynamics of the evolution dominates once again. We note that the Einsteins static universe solution permitted here is unstable which the asymptotic EU might follow. We also compare our EU model with supernova data.
\end{abstract}

\footnotetext{
*bcpaul@iucaa.ernet.in

†souviknbu@rediffmail.com
} 


\section{Introduction:}

Recent cosmological observations predict that the universe is passing through an accelerated phase of expansion [1]. Although the Einsteins General theory of Relativity (GTR) with usual matter fields of the standard model of particle physics admits early inflation fairly well [2, 3], it fails to accommodate the recent accelerating phase. This led in modern cosmology to explore a suitable theory which accommodates observational prediction. Perhaps the present accelerating phase of expansion may be realized either modifying the matter sector or the gravitational sector including higher derivative terms that are relevant in the low curvature limit. It is generally accepted that inflation is an essential ingredient to construct cosmological models in modern cosmology. The early inflation can be realized consistently in a semi-classical theory of gravity [3, 4]. It may be mentioned here that Starobinsky [5] obtained inflationary solution in a higher derivative theory of gravity long before the advent of inflation actually realized. The gravitational action of the Starobinsky model corresponds to a theory which contains curvature squared terms in the the EinsteinHilbert action. However, the efficacy of inflation is known only after the seminal work of Guth [3] who used phase transition mechanism to obtain such scenario in order to resolve some of the outstanding problems of Big bang model. The idea of inflation is very much attractive which has been implemented in various theories leading to a number of versions of inflation in the literature. However, the late accelerating phase is very recently predicted from observational data and a suitable explanation is yet to come out. To address the issue a number of proposals came up (i) with a suitable modification of the matter sector by incorporating exotic kind of matter fields [6] or (ii) with a modification in the gravitational sector by adding curvature squared or its inverse power terms that are effective at low curvature limit [7] to the Einstein-Hilbert action. It has been shown [7] that the present

accelerating phase of the universe may be obtained by adding a $\frac{\mu^{4}}{R}$ term to the Einstein-Hilbert action. However, Einstein-Hilbert action with the inverse curvature correction term only is not enough as it is not free from shortcomings. Later, it has been shown that the above shortcomings of the theory may be removed by adding a polynomial in Ricci scalar i.e., curvature squared term to the proposed gravitational action [8]. It was shown long ago by Zweibach [9] that the string corrections due to Einstein action up to first order in the slope parameter 
and fourth power of momenta should be proportional to Gauss-Bonnet (GB) terms (where $G B=R_{\mu \nu \gamma \delta} R^{\mu \nu \gamma \delta}-4 R_{\mu \nu} R^{\mu \nu}+R^{2}$ ). However, it was realized subsequently that the field redefinition theorem of 't Hooft and Veltman [10] may be applicable in this case. On the Einstein's shell $\left(R_{\mu \nu}=0\right)$, an action with curvature squared term of the form $R+a R_{\mu \nu}^{2}+b R^{2}$ may be transformed into $R$ itself (neglecting higher order terms) by field redefinition :

$$
g_{\mu \nu}^{\prime}=g_{\mu \nu}+a R_{\mu \nu}+g_{\mu \nu} \frac{a+2 b}{2-D} R
$$

where $D$ represents the number of dimensions. Subsequently Deser and coworkers [11 have shown that on the linearized Einstein shell, the actions $R+\alpha^{\prime}(G B)$ and $R+\alpha^{\prime} R_{\mu \nu \gamma \delta}^{2}$ (here $\alpha^{\prime}$ is the inverse of string tension) are not different and this result generalizes to all higher-order ghost terms. GB terms arise naturally as the leading order of the $\alpha^{\prime}$ expansion of heterotic superstring theory [12]. It is known that GBterms in the higher dimensions leads to ghost free propagator. It may be mentioned here that in the framework of 4 dimensions, the GB terms do not contribute in the dynamics of evolution. However, GB terms if coupled with a dilaton field in the action then the combination plays an important role in the dynamics of the evolution via the dilaton field. It has been shown also that GB combinations coupled with scalar field plays an important role for avoidance of singularity in a string induced gravity [13. Later, in the braneworld scenario, it has been shown [14 that the naked singularities may not occur if dilaton with a Gauss-Bonnet term are considered. The issues of fine tunning in a theory with a dilaton field and GB-terms interaction are also taken into account to study in details in Ref. [14, 15]. Recently, cosmological models with dark energy of the universe are probed in the Einstein-Hilbert action with GB term and it is known that the theory accommodates the new form of energy [16]. It has been shown [17] that scalar field that enters into the coupling of GB terms in the action plays an important role which may be used to explain different phases of expansion of the universe including the present accelerating phase. It is also shown that GB terms with dilaton admits accelerating cosmologies in the framework of higher dimensions [19]. The GaussBonnet terms in the Einstein-Hilbert action are used to obtain new black holes solution [18] and Kaluza-Klein space-times [21, 22].Paul and Mukherjee [24] earlier noted that a Gauss-Bonnet term in higher dimensions leads to 3 a 4-dimensional universe at a later epoch with 
many good features considering the sign of the coupling parameter different from that one usually gets from the low energy limit of string theory. The model also gives a satisfactory explanation of the smallness of the effective four dimensional cosmological constant. Recently Einstein-Hilbert action with a combination of higher order curvature terms e.g., GB terms including dilaton are employed to study the present acceleration of the universe [17, 18]. Therefore, the GB-theory has a rich structure that needs to be explored. In this paper we explore emergent universe scenario in the Einstein-Hilbert action with GB terms coupled with dilaton field in the frame work of four dimensions. We employ the GB term with dilaton in four dimensions to obtain an emergent universe scenario [24, 25].

Earlier Harrison [26] obtained a cosmological solution with radiation in the presence of a cosmological constant in a closed model of the universe which asymptotically approaches to Einstein static universe but the scenario does not exit inflationary phase. Recently, Ellis and Maartens [27] obtained similar cosmological solution considering a minimally coupled scalar field with a special choice of its potential where the universe exits from its inflationary phase followed by reheating. Subsequently it was shown by Ellis et al. 23] that the potential required to obtain such scenario may be obtained naturally by a conformal transformation of Einstein-Hilbert action with $R^{2}$-term for a proper choice of its coupling constant. The model incorporates an asymptotically Einstein static universe in the past and it evolves to an accelerating universe in the framework of a closed model of the universe. This model is usually known as emergent universe model. The salient features of an emergent universe scenario is that there is no time like singularity, it is ever existing and it approaches a static universe in the infinite past $(t \rightarrow-\infty)$. It is interesting to construct an emergent universe model as it is capable of solving some conceptual issues of the standard Big bang model. The asymptotic Einstein static universe at some stage enters into the standard Big bang phase and might have features precisely known to us. The possibilities of an emergent universe scenario have been studied recently in a number of theories [24, 25, 27, 28, 29, 30, 31, 32, 33, 34 because it permits a universe which is ever existing and large enough so that the spacetime may be treated as classical entities. Recently, Mukherjee et al. 24] obtained an emergent universe (EU) scenario in a flat universe in the modified Starobinsky model. Subsequently, Mukherjee et al. 25] proposed a general framework for such an EU scenario in GR with 
a mixture of matter and exotic kinds of matter that are prescribed by an equation of state (EOS) : $p=A \rho-B \sqrt{\rho}$, where $A$ and $B$ are constants. The EU scenario can be realized for some possible soup comprising primordial compositions of matter that are permitted by the EOS. It admits existence of exotic matter and dark energy in addition to radiation/dust [25]. Since recent cosmological observations indicate that our universe is almost flat, the above emergent model of the universe is explored in a flat universe context. Subsequently the EU scenario proposed by Mukherjee et al. [24, 25] are explored in the context of various theories and found that the scenario can be realized fairly well [31, 32, 33]. The purpose of the paper is to examine EU scenario in a gravitational action with GB-terms coupled with a dilaton field. The plan of the paper is as follows: in sec. 2, we present the gravitational action and set up the relevant field equations, in sec. 3 , cosmological solutions with emergent universe scenario are derived. Finally, in sec. 4, we summarize the results obtained.

\section{Action and the Field Equations:}

We consider a gravitational action given by

$$
I=-\int\left[\frac{R}{2 \kappa^{2}}+f(\phi)\left(R_{\mu \nu \gamma \delta} R^{\mu \nu \gamma \delta}-4 R_{\alpha \beta} R^{\alpha \beta}+R^{2}\right)+L_{\phi}\right] \sqrt{-g} d^{4} x
$$

where the Greek indices $\mu, \nu$ represents $(0,1,2,3), f(\phi)$ represents the coupling factor of the Gauss-Bonnet (GB) term and GB is a combination of squared terms of Riemann tensor, Ricci tensor and Ricci scalar $\left(G B=R_{\mu \nu \gamma \delta} R^{\mu \nu \gamma \delta}-4 R_{\alpha \beta} R^{\alpha \beta}+R^{2}\right), g$ represents the 4 dimensional metric, $8 \pi G=\kappa^{2}$ and $L_{\phi}$ represents the Lagrangian for the dilaton field. The corresponding Lagrangian for the dilaton field is given by

$$
L_{\phi}=-\xi(\phi) \partial_{\mu} \phi \partial^{\mu} \phi-V(\phi)
$$

where $\xi(\phi)$ represents the coupling parameter for the field in the gravitational action and $V(\phi)$ represents potential of the dilaton field.

We consider a flat, homogeneous and isotropic Robertson-Walker (RW) metric with scale factor $a(t)$, which is given by

$$
d s^{2}=-d t^{2}+a^{2}(t)\left[d r^{2}+r^{2}\left(d \theta^{2}+\sin ^{2} \theta d \phi^{2}\right)\right] .
$$


The action given in (1) with the RW metric (3) yields the following field equations :

$$
\begin{gathered}
3\left(\frac{\dot{a}}{a}\right)^{2}=\kappa^{2}\left[\xi(\phi) \dot{\phi}^{2}+V(\phi)-24 f^{\prime}(\phi) \dot{\phi} \frac{\dot{a}^{3}}{a^{3}}\right] \\
2 \frac{\ddot{a}}{a}+\left(\frac{\dot{a}}{a}\right)^{2}=-\kappa^{2}\left[\xi(\phi) \dot{\phi}^{2}-V(\phi)+16 f^{\prime}(\phi) \dot{\phi} \frac{\dot{a} \ddot{a}}{a^{2}}+8\left(f^{\prime}(\phi) \ddot{\phi}+f^{\prime \prime}(\phi) \dot{\phi}^{2}\right) \frac{\dot{a}^{2}}{a^{2}}\right]
\end{gathered}
$$

once again varying the action (2.1) with respect to the dilaton field $\phi$, we get

$$
\xi(\phi)\left[\ddot{\phi}+3 \frac{\dot{a}}{a} \dot{\phi}+\frac{1}{2} \frac{\xi^{\prime}}{\xi} \dot{\phi}^{2}+\frac{V^{\prime}(\phi)}{2 \xi}\right]=12 f^{\prime}(\phi) \frac{\dot{a}^{2} \ddot{a}}{a^{3}}
$$

where the over dot implies derivative w.r.t. time and prime $\left(^{\prime}\right)$ represents differentiation with respect to the field $\phi$. In the above, out of the three eqs. (4)-(6), only two are independent, as eq. (2.6) can be derived from eqs. (4) and (5). It is evident that there are altogether five unknowns namely, $a(t), \phi, V(\phi), \xi(\phi)$ and $f(\phi)$ in the above field equations. In order to solve the equations additional assumptions are can be made. Let us first assume that

$$
f^{\prime}(\phi) \dot{\phi}=\eta
$$

where $\eta$ is a constant. The above assumption leads to a relation $f(\phi)=\eta t(\phi)+\eta_{o}$, where $\eta_{o}$ is a constant. The coupling parameter $f(\phi)$, therefore, grows with time. Consequently the effect of the GB terms becomes more and more important at late time, which may be useful for describing the present acceleration of the universe. Using the constraint given by eq. (7) in eqs. (4) and (5), one gets

$$
\begin{gathered}
3 H^{2}=\kappa^{2}\left[\xi(\phi) \dot{\phi}^{2}+V(\phi)-24 \eta H^{3}\right], \\
2 \dot{H}+3 H^{2}=-\kappa^{2}\left[\xi(\phi) \dot{\phi}^{2}-V(\phi)+16 \eta H\left(\dot{H}+H^{2}\right)\right],
\end{gathered}
$$

where $H=\frac{\dot{a}}{a}$ is the Hubble parameter. Now eliminating $V(\phi)$ from eqs. (8) and (9), we get

$$
\left.\dot{H}+\kappa^{2}\left[\xi(\phi) \dot{\phi}^{2}+8 \eta H \dot{H}-4 \eta H^{3}\right)\right]=0 .
$$


The dilaton potential can be obtained from eqs. (8) and (9) eliminating $\xi(\phi) \dot{\phi}^{2}$, the corresponding potential becomes a function of Hubble parameter which is given by

$$
V(H)=\frac{3}{\kappa^{2}} H^{2}+20 \eta H^{3}+\frac{1}{\kappa^{2}}\left(1+8 \eta \kappa^{2} H\right) \dot{H},
$$

the above potential may be expressed as a function of $\phi$ once the Hubble parameter $(H)$ is known in terms of $\phi$, which will be determined in the next section. We use eqs.(8)-(9) to determine the coupling parameters $\xi(\phi)$ and $f(\phi)$ in the theory for an EU cosmological solution following Ref. [17]. The set of eqs. (10)-(11) contain four unknowns, therefore, two more ad hoc assumptions can be made to obtain a consistent cosmological solution. In the next section we begin with a known scale factor, which permits an emergent universe scenario.

\section{Cosmological Solutions}

Let us consider the evolution of the scale factor of the universe in the form

$$
a(t)=a_{o}\left[A+e^{\alpha t}\right]^{\frac{1}{\beta}}
$$

where $a_{o}, \alpha, \beta$ and $A$ are positive constants. It gives an EU scenario as have been obtained in Refs. [24, 25]. The Hubble parameter corresponding to eq.(12) satisfies a first order differential equation given by

$$
\dot{H}=\alpha H-\beta H^{2} .
$$

From eqs. (8)-(10), the dilaton coupling and the dilaton potential are now can be determined. The field equations are highly non-linear. Consequently it is not simple to obtain a general form of $\xi(\phi)$ and $V(\phi)$ in terms of the dilaton field. However, those parameters may be determined in terms of the Hubble parameter following Ref. [17, which are

$$
\begin{aligned}
& \xi(H)=\frac{1}{\kappa^{2} \dot{\phi}^{2}}\left[4 \eta \kappa^{2}(1+2 \beta) H^{3}+\left(\beta-8 \kappa^{2} \alpha \eta\right) H^{2}-\alpha H\right], \\
& V(H)=\frac{3}{\kappa^{2}} H^{2}+20 \eta H^{3}+\frac{1}{\kappa^{2}}\left(1+8 \kappa^{2} \eta H\right)\left(\alpha H-\beta H^{2}\right) .
\end{aligned}
$$

For simplicity, as a special case let us consider $\beta=8 \eta \kappa^{2} \alpha$ in the above.the corresponding dilaton field potential can be expressed in 
terms of the field. There is another freedom to assume here as the number of unknowns are one more than the number of relevant equations. Therefore, we look for emergent universe scenario for different behaviors of the dilaton field in the next subsection.

\subsection{Case I :}

For an increasing dilaton field $\phi=\phi_{o} e^{\alpha t}$, with the GB coupling terms $f(\phi)=\frac{\eta}{\alpha} \ln \phi$, dilaton coupling is given by

$$
\xi(\phi)=\frac{1}{2 \kappa^{2} \phi(1+\beta \phi)^{3}}\left(\beta \phi^{2}-4 \beta \phi-2\right)
$$

We note the following: $(i) \xi(\phi) \rightarrow \infty$ when $\dot{a} \rightarrow 0$ i.e. $H \rightarrow 0$ and $(i i) \xi(\phi) \rightarrow 0$ at two points (a) for $H_{1}=\frac{\alpha}{\beta}$ and (b) for $H_{2}=$ $\frac{2 \alpha^{2}}{\beta(1+2 \beta)}$. We plot the variation of the dilaton coupling $(\xi)$ with $\phi$ as shown in the fig.1. It is evident that initially the dilaton coupling begins with negative value (phantom like property) but in course of its evolution $\xi(\phi)$ becomes positive and almost constant after attaining a peak. Thus in this model to begin with one can start with a field having negative kinetic energy, an interesting field which behaves like phantom [35], now-a-days it is considered as one of the candidate of dark matter and avoids the singularity. The potential is given by

$$
V(\phi)=\frac{\alpha^{2}}{\kappa^{2}(1+\beta \phi)^{3}}\left(\frac{11}{2} \beta \phi^{3}+(2 \beta+3) \phi^{2}+\phi\right) .
$$

The Hubble parameter in this case is related to field as

$$
H=\frac{\alpha \phi}{1+\beta \phi} \text {. }
$$

We determine $A=1, \phi_{o}=\frac{1}{\beta}$ and the corresponding evolution of the scale factor becomes

$$
a=a_{o}\left[1+e^{\alpha t}\right]^{\frac{1}{\beta}} .
$$

\subsection{Case II :}

For a decreasing dilaton field $\phi=\phi_{o} e^{-\alpha t}$, with the GB coupling term $f(\phi)=-\frac{\eta}{\alpha} \ln \phi$, the dilaton coupling is given by

$$
\xi(\phi)=\frac{1}{2 \kappa^{2} \phi^{2}(\beta+\phi)^{3}}\left(\beta-4 \beta \phi-2 \phi^{2}\right)
$$




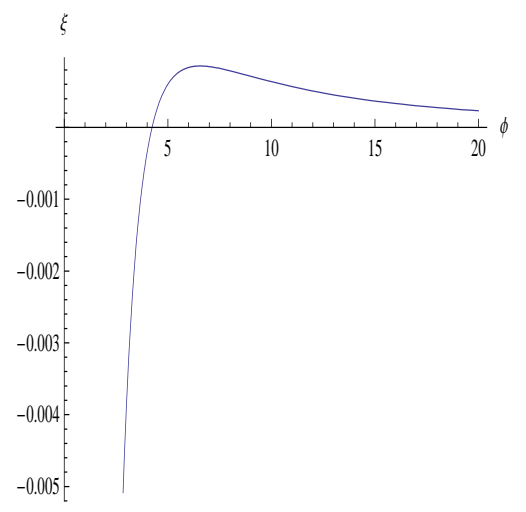

Figure 1: $\xi(\phi)$ Vs. $\phi$ curve for $\kappa=1$ and $\beta=2$

In fig. 2 we plot variation of dilaton coupling $\xi(\phi)$ with $\phi$. It is evident that $\xi(\phi)$ begins with a negative value then attains a minimum thereafter it increases as $\phi$ decreases. The potential for the dilaton field is obtained as

$$
V(\phi)=\frac{\alpha^{2}}{\left.\kappa^{2}(\beta+\phi)^{3}\right)}\left(\frac{11}{2} \beta+(2 \beta+3) \phi+\phi^{2}\right) .
$$

The Hubble parameter is given in terms of the dilaton as

$$
H=\frac{\alpha}{\beta+\phi}
$$

the corresponding scale factor is

$$
a=a_{o}\left[\phi_{o}+\beta e^{\alpha t}\right]^{\frac{1}{\beta}} .
$$

\subsection{Case III :}

For a slowly varying field $\phi=\frac{1}{\alpha} \ln t$, with $f(\phi)=\eta e^{\alpha \phi}$, similar to that one expects in the string theory framework [34], the dilaton coupling is given by

$$
\xi(\phi)=\frac{1}{\kappa^{2}}\left[\frac{\beta(1+2 \beta)}{2 \alpha} H^{3}-\alpha H\right]\left(\ln \frac{H}{\alpha-\beta H}\right)^{2},
$$

and the dilaton potential is

$$
V(H)=\frac{1}{\kappa^{2}}\left(\alpha H+3 H^{2}+\frac{\beta}{\alpha}\left(\frac{5}{2}-\beta\right) H^{3}\right)
$$




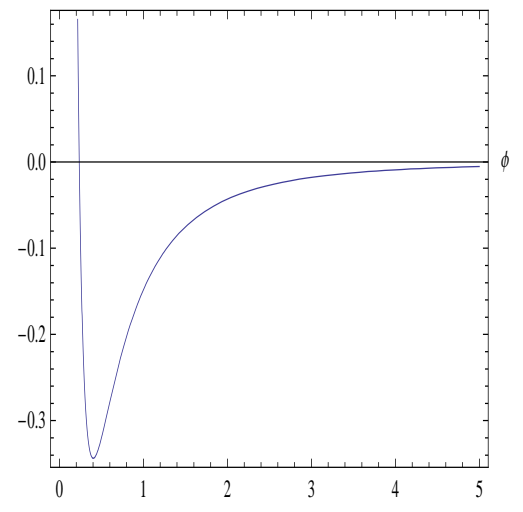

Figure 2: $\xi(\phi)$ vs. $\phi$ curve for $\kappa=1$ and $\beta=2$

where the Hubble parameter $(\mathrm{H})$ is given by

$$
H=\frac{\alpha e^{\alpha e^{\alpha \phi}}}{1+\beta e^{\alpha e^{\alpha \phi}}} .
$$

Fig. 3 shows variation of $\xi(\phi)$ with $\phi$ for $\beta=5$. Here the dilaton coupling decreases as the dilaton increases to begin with from a negative value initially, attains a minimum, thereafter, it increases sharply. GB terms become important at late time in this case. The potential becomes flat as $\phi \rightarrow \infty$ which is shown in fig. 4 .

\subsection{Case IV :}

For a dilaton field $\phi=H$, considering variation of the coupling of GB term as $f=\eta t$. We obtain :

$$
\xi(\phi)=\frac{1}{\kappa^{2} \phi^{2}(\alpha-\beta \phi)^{2}}\left[\frac{\beta(1+2 \beta)}{2 \alpha} \phi^{2}-\alpha\right]
$$

with dilaton potential given by

$$
V(\phi)=\frac{1}{\kappa^{2}}\left(\alpha \phi+3 \phi^{2}+\frac{\beta(5-2 \beta)}{2 \alpha} \phi^{3}\right)
$$

The fig. 5 shows the variation of $\xi(\phi)$ vrs. $\phi$, for $\beta=5$. We note that the coupling parameter becomes undetermined when $\phi=\frac{\alpha}{\beta}$. In this case it is necessary to begin with an initial field which greater than the above limiting value. In that case the coupling parameter 


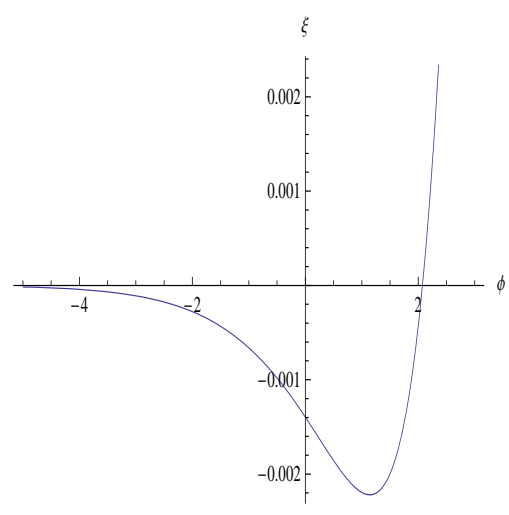

Figure 3: $\xi(\phi)$ Vs. $\phi$ curve for $\beta=5$

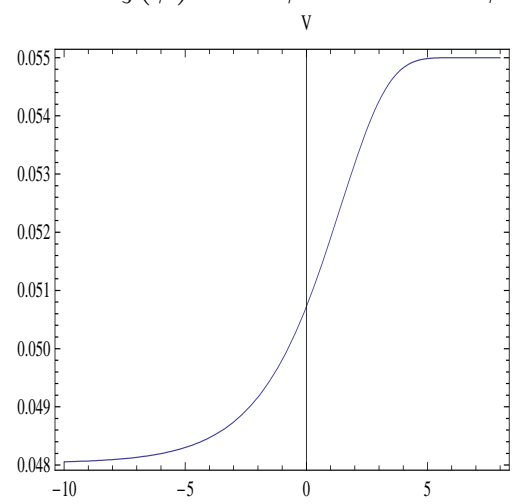

Figure 4: $V(\phi)$ vs. $\phi$ curve for $\alpha=0.5$ and $\beta=5$ 
always remains positive definite and it decreases from a large value to zero. Here the GB terms do not contribute at late time as the coupling $\xi \rightarrow 0$. The corresponding dilaton potential required for the EU model is shown in fig. 6. It has a minimum which is negative definite.The dilaton potential for $\beta=\frac{5}{2}$, becomes

$$
V(\phi)=\frac{3}{\kappa^{2}}\left(\phi+\frac{\alpha}{6}\right)^{2}-\frac{\alpha^{2}}{12 \kappa^{2}} .
$$

In this case the universe evolves as

$$
a=a_{o}\left(1+\frac{5}{2} e^{\alpha t}\right)^{\frac{2}{5}}
$$

\subsection{Case V :}

We now consider a special case $f(\phi)=\eta_{o}+\eta \phi^{2}$ to obtain EU scenario. In this case the dilaton evolve as $\phi= \pm \sqrt{t}$ with the coupling parameter

$$
\xi(\phi)=\frac{4 \phi^{2}}{\kappa^{2}}\left(-\alpha H+\frac{\beta}{2 \alpha}(1+2 \beta) H^{3}\right) .
$$

The dilaton field potential is

$$
V(\phi)=\frac{1}{\kappa^{2}}\left(\alpha H+3 H^{2}+\frac{\beta}{\alpha}\left(\frac{5}{2}-\beta\right) H^{3}\right)
$$

where $H=\frac{\alpha e^{\alpha \phi^{2}}}{1+\beta e^{\alpha \phi^{2}}}$.

Fig. 7 shows the variation of the dilaton coupling parameter with field, which is interesting. The variation of the potential is also shown in fig. 8, which has two flat regions one in the early era and the other in the late era respectively. This is new and interesting as it permits both early inflation and late acceleration. In Ref. [24] it was shown that a particle creation may occur during a phase when the Hubble parameter varies slowly. In this case the GB coupling parameter $f(\phi) \rightarrow \pm \infty$ as $t \rightarrow \pm \infty$. The GB combination might have dominated in the early epoch of evolution which eventually decreases at later epoch (for $\eta_{o}=0$ ) corresponding to a minimum of $f(\phi)$ (say at $t=0$ ) thereafter it increases which is shown in fig. 9. Thus GB terms play an important role in the early era which is subsequently important once again at late era contributing to the dark energy [8]. 


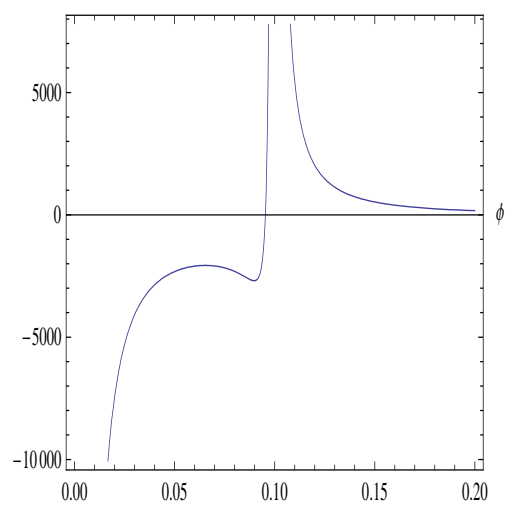

Figure 5: $\xi(\phi)$ vs. $\phi$ curve for $\alpha=0.5$ and $\beta=5$

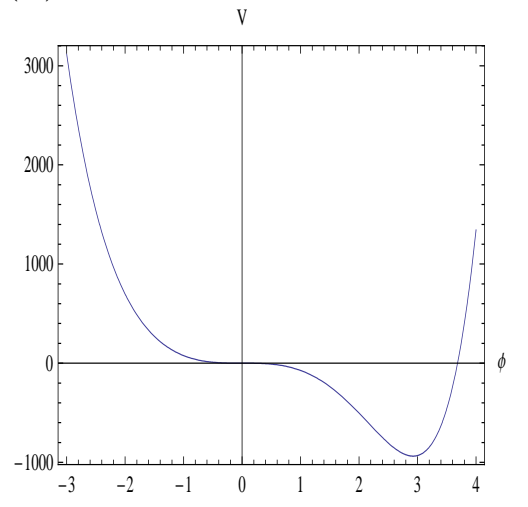

Figure 6: $V(\phi)$ vs. $\phi$ curve for $\alpha=0.5$ and $\beta=5$ 


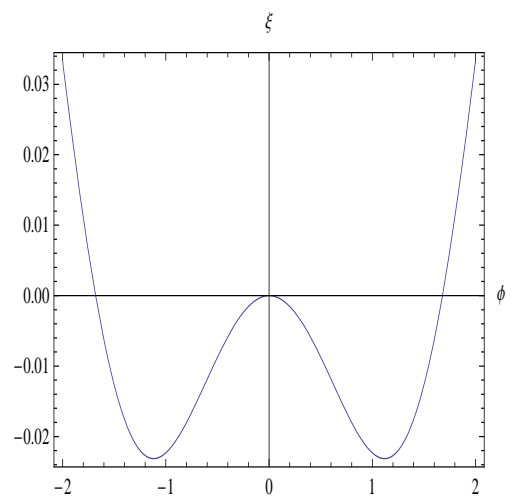

Figure 7: $\xi(\phi)$ vs. $\phi$ curve for $\beta=5$

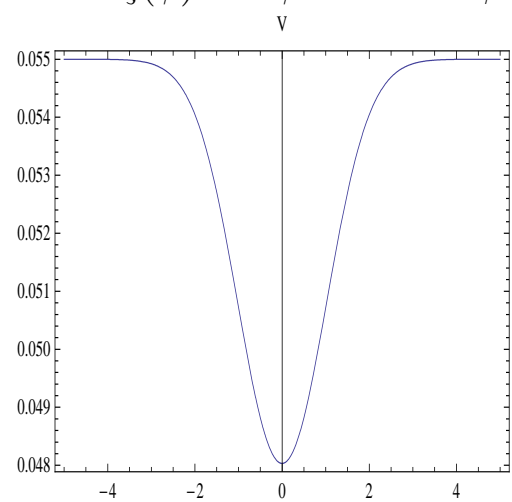

Figure 8: $V(\phi)$ vs. $\phi$ curve for $\beta=5$

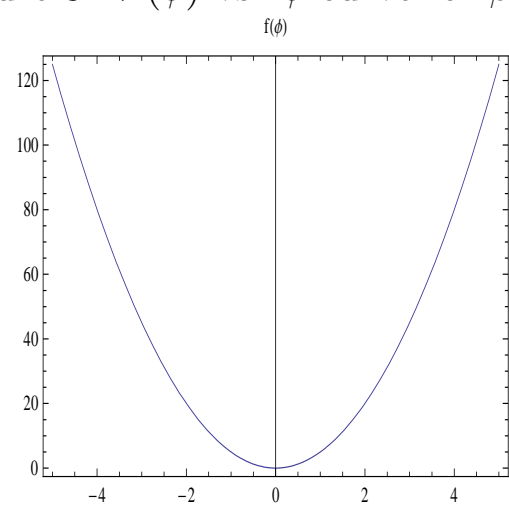

Figure 9: $f(\phi)$ vs. $\phi$ curve for $\eta_{o}=0$ and $\eta=5$ 


\section{Stability of Einstein's Static Universe}

The EU scenario is characterized by the scale factor $a(t)=\left(a_{0}+\right.$ $\left.\beta e^{\alpha t}\right)^{1 / \beta}$ as given in (12). It may be pointed out here that the universe (i) begins with singularity if $\beta<0$, (ii) begins with singularity and asymptotically approaches Einstein static (ES) universe at late time if $\alpha<0$ and $\beta \neq 0$, and (iii) spends infinite time near the Einstein static universe but pulls away and ends in an infinite inflating epoch if $\alpha>0$ and $\beta>0$. It is pointed out that the first two cases lead to unstable solution [37]. It may be pointed out here that: (i) The existence of the ES universe in fourth order theories of gravity and stability of the ES has been studied in [39]. (ii) Stability of ES in $f(R)$ gravity has also been considered in [40]. (iii) It was also noted that ES universes are unstable in generic $f(R)$ models [41]. To analyze the stability of Einsteins Static (ES) universe (which is an asymptotic past solution) [38] in the theory we begin with a pair of differential equations in $a$ and $H$ which are given by

$$
\begin{gathered}
\dot{a}=a H . \\
\dot{H}=\alpha H-\beta H^{2},
\end{gathered}
$$

The above equations form an autonomous system which can be analyzed by the standard technique. The Einstein static universe solution corresponds to the critical point of the system $\left(a_{0}, 0\right)$. The ES universe in this case is unstable for $\alpha>0$. It may be pointed out here that the stability of the Einstein Static universe under inhomogeneous perturbations has recently been studied in Refs. [42].

\section{Distance modulus curve:}

We now probe late universe in the Emergent Universe scenario taking into account the observational results available from supernova. The distance modulus is $\mu=5 \log d_{L}+25$ where $d_{L}$ is the luminosity distance (in the unit of mega parsecs), given by

$$
d_{L}=r_{1}(1+z) a\left(t_{0}\right)
$$

where $r_{1}$ is given by :

$$
\int_{0}^{r_{1}} \frac{d r}{\left.\sqrt{1-k r^{2}}\right)}=\int_{t_{1}}^{t_{0}} \frac{d t}{a(t)}
$$




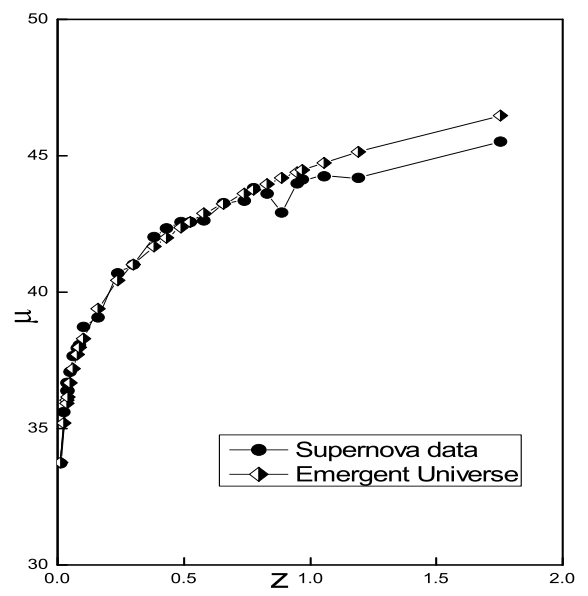

Figure 10: $\mu$ vs. $z$ curve for Emergent universe and supernova data.

We consider the scale factor for a EU scenario as was given in (12),

$$
a(t)=a_{0}\left(\sigma+e^{\alpha t}\right)^{\omega},
$$

where $a_{0}, \sigma$ and $\omega$ are constants. At late time the exponential term dominates and one can write

$$
a(t) \sim a_{0} e^{\alpha \omega t}
$$

Since we are considering a flat universe, $k=0$, eq. (36) yields

$$
r_{1}=\int_{t_{1}}^{t_{0}} \frac{d t}{a(t)}
$$

Using the scale factor (37) one obtains an expression for $\mathrm{dL}$ which is given by

$$
d_{L}=\frac{z(1+z)}{H_{0}}
$$

Note that the final expression for $d_{L}$ does not depend on $a_{0}, \sigma$ and $\omega$. It is now possible to determine $\mu(z)$ numerically for an Emergent Universe scenario at different values of redshift parameters $(z)$. The observed values of $\mu(z)$ at different $z$ parameters [1] along with that obtained from the present theory are given in Table : 1. 


\begin{tabular}{|c|c|c|}
\hline$z$ & Supernova $\mu$ & $E U \mu$ \\
\hline 0.038 & 36.67 & 36.0438240 \\
0.014 & 33.73 & 33.7609468 \\
0.026 & 35.62 & 35.1945228 \\
0.036 & 36.39 & 35.9222306 \\
0.040 & 36.38 & 36.1593860 \\
0.050 & 37.08 & 36.6647158 \\
0.063 & 37.67 & 37.1932883 \\
0.079 & 37.94 & 37.7171620 \\
0.088 & 38.07 & 37.9694771 \\
0.101 & 38.73 & 38.2944627 \\
0.160 & 39.08 & 39.4068091 \\
0.240 & 40.68 & 40.4320839 \\
0.300 & 41.01 & 41.0192423 \\
0.380 & 42.02 & 41.6622327 \\
0.430 & 42.33 & 42.0079418 \\
0.490 & 42.58 & 42.3808310 \\
0.526 & 42.56 & 42.5866208 \\
0.581 & 42.63 & 42.8794593 \\
0.657 & 43.27 & 43.2483587 \\
0.740 & 43.35 & 43.6128241 \\
0.778 & 43.81 & 43.7684760 \\
0.828 & 43.61 & 43.9639519 \\
0.886 & 42.91 & 44.1787963 \\
0.949 & 43.99 & 44.3993095 \\
0.970 & 44.13 & 44.4701090 \\
1.056 & 44.25 & 44.7473544 \\
1.190 & 44.19 & 45.1438747 \\
1.755 & 45.53 & 46.4859129 \\
\hline
\end{tabular}

\section{Discussion}

We obtain emergent universe scenario in a modified theory of gravity with Gauss-Bonnet term coupled to a dilaton field in four dimensions. We look for an emergent universe (EU) scenario here in a spatially flat universe. In the EU model, the universe was in an almost static state in an infinite past which eventually evolves into an inflationary stage later on. The Einstein static universe permitted here is found unstable. We note that emergent universe scenario obtained in Refs. [24, 25] can be implemented in a modified theory of gravity with GB 
terms quite successfully with a suitable dilaton and GB coupling parameters. The parameters are determined here and the corresponding models are presented in cases $\mathrm{I}$ to $\mathrm{V}$ in section 3 . The result obtained here once again supports the view that the GB combination might have played a crucial role for driving both early inflation and late acceleration. A new solution is noted here in which the coupling parameter of the GB terms becomes important in the early and late era. This is an interesting solution with a new dilaton potential which is shown in fig. 8. The late acceleration of the universe may be explained in this framework quite successfully as is shown in fig. 10 by the distance modulus curve. We also note cosmological solution where the dilaton field behaves like phantom [35] in the early era but it transits to non-minimally coupled scalar field in the later epoch in all the cases except in one case, where $\phi=H$ and $f(\phi)$ increases linearly with time. However it is evident from fig. 6 that there is a regim where $V(\phi)$ remains negative. We compare our Emergent Universe model recent SNeIa data [1] at late universe. Fig. 10 show a comparative study of $\mu(z)$ vs. $z$ curve obtained from observation and that obtained theoretically from Emergent universe model. It may be pointed out here that between an exponential and accelerating phase, there might exist a phase of particle creation era which is pointed out in Ref. [24] in the context of EU. However, a detail study on the issue will be taken up elsewhere.

\section{Acknowledgments}

BCP would like to thank Prof. S. Randjbar-Daemi for supporting ICTP visit where this work was initiated and Third World Academy of Sciences (TWAS) for awarding visiting Associateship to visit Institute of Theoretical Physics, Beijing Peoples Republic of China. BCP would also like to thank UGC for awarding Minor Research Project grant (No. F. 32-63/2006 (SR)). SG likes to thank IUCAA Reference Centre at North Bengal University for extending facilities to do research work and the University of North Bengal for awarding Junior and Senior Research Fellowship. The authors are thankful to the referee for his constructive suggestions which helped in improving the presentation of the paper. 


\section{References}

[1] A. G. Riess et al., Astron. J. 607665 2004, arXiv : astroph/ 0402512; S. Perlmutter et al., Nature 51391 (1998); S. Perlmutter et al., Astron. J. 598102 (2003); S. Perlmutter et al., Astron. J. 517565 (1999); P. de Bernardis et al., Nature 404 955 (2000); M. Tegmark et al., Phys. Rev D69 103501 (2004); D. N. Spergel et al., astro-ph/0603449.

[2] S. W. Weinberg, Gravitation and Cosmology( New York, Wiley 1972); E. W. Kolb and M. S. Turner, The Early Universe (New York, Addison-Wesley, 1990); A. D. Linde, Particle Physics and Inflationary Cosmology (New York, Gordon and Breach 1990); L. F. Abbot and S. Y. Pi, Inflationary Cosmology, (Singapore, World Scientific 1986).

[3] A. H. Guth, Phys. Rev. D23 347 (1981).

[4] A. D. Linde, Phys. Lett. B108 389 (1982); Rep. Prog. Phys. 47 925 (1984); R. H. Brandenberger, Rev. Mod. Phys. 571 (1985); D. H. Lyth, D. Roberts and M. Smith, Phys. Rev. D57 7120 (1998); L. Mersini, Mod. Phys. Lett. A16 1933 (2001); A. Linde, Int. J. Mod. Phys. A17 89 (2002).

[5] A. A. Starobinsky, Phys. Lett. B99 24 (1980).

[6] X.-H Meng, M. Hu and J. Ren, astro-ph/0510357; X. Zhai, Y. $\mathrm{Xu}$ and X. Li, Int. J. Mod. Phys. D15 1151 (2006); A. A. Sen and R. T. Scherrer, Phys. Rev. D72 063511 2005; Z. K. Guo and Y. Z. Zhang, Phys. Lett. B645 326 (2007); O. Bertolami, astro-ph/0504275; H. B. Benaoum, hep-th/0205140; W. Chakraborty and U. Debnath, gr-qc/0611094; gr-qc/0705.4147; D. A. Steer and F. Vernizzi, Phys. Rev. D70 043527 2004; N. Cruz, S. Lepe and F. Pena, gr-qc/0609013; M. R. Setere, Phys. Lett. B648 329 2007; P. Wu and H. Yu, Phys. Lett. B644 16 (2007); J. A. Jim'nez Madrid, Phys. Lett. B634 106 (2006).

[7] S. M. Carroll, V. Duvvuri, M. Trodden and M. S. Turner, Phys. Rev D70 043528 (2004), astro-ph/0306438; S. Capozzielo, S. Carloni and A. Troisi, Recent Res. Dev. Astron. Astrophys. 1625 (2003).

[8] S. D. Nojiri and S. D. Odintsov, Phys. Lett. B 5765 (2003); S. D. Nojiri and S. D. Odintsov, Phys. Rev. D 68123512 (2003); M. 
Abdalla, S. D. Nojiri and S. D. Odintsov, Class. Quant. Grav. 22135 (2003); I. Brevik, S. D. Nojiri and S. D. Odintsov and I. Vanzo, Phys. Rev. D70 043520 (2004); S. Capozzielo, V. F. Cardone and A. Troisi, Phys. Rev. D71 043503 (2005).

[9] B. Zwiebach, in Anomalies, Geometry and Topology, proceedings of the Symposium, Argonne, Illinois, 1985, edited by W. A. Bardeen and A. White (World Scientific, Singapore, 1985); Phys. Lett. 156B 315 (1985).

[10] G. t Hooft and M. Veltman, Ann. Inst. Henri Poincar 2069 1974; G. t Hooft, in Functional and Probabalistic Methods in Quantum Field Theory, proceedings of the XII Winter School on Theoretical Physics, Karpacz, Poland, 1975, edited by B. Jancewicz (Wydawnictwa Uniwersytetu Wroclawskiego, Wroclaw, 1976).

[11] S. Deser, presented at the Second Nobel Symposium on Elementary Particle Physics, Marstrand, Sweden, 1986 (unpublished); S. Deser, Phys. Scr. T 15138 (1987); N. Deruelle and J. Madore, Phys. Lett. B 18625 (1987); S. Deser and A. N. Redlich, Phys. Lett. B 176350 1986; D. G. Boulware and S. Deser, Phys. Lett. B 175409 1986; J. Madore, J. T. Wheeler, Nucl. Phys. B 268 737 1986; N. Deruelle and J. Madore, Phys. Lett. A 114185 (1986); D. G. Boulware and S. Deser, Phys. Rev. Lett. 552656 (1985); D. Lovelock, J. Math. Phys. 12498 (1971).

[12] J. Callan et al. Nucl. Phys. B 262593 (1985); D. J. Gross and J. H. Sloan, Nucl. Phys. B 29141 (1987); R. R. Metsaev and A. A. Tseytlin, Phys.Lett. B 191, 354 (1987); M. C. Bento and O. Bertolami, Phys.Lett. B 368, 198 (1995).

[13] I. Antoniadis, J. Rizos and K. Tamvakis, Nucl. Phys. B415 497 (1994).

[14] P. Binetruy, C. Charmousis, S. C. Davis and J-F. Dufaux, Phys. Lett. B544 183 (2002), hep-th/0206089.

[15] N. E. Mavromatos and J. Rizos, Phys. Rev D62 124004 (2000), Int. J. Mod. Phys. A18 57 (2003); A. Jakobek, K. A. Meissner and M. Olechowski, Nucl. Phys. B645 217 (2002).

[16] S. D. Nojiri and S. D. Odintsov and M. Sasaki,, Phys. Rev. D71 123509 2005; S. D. Nojiri and S. D. Odintsov, Phys. Lett. B631 1 (2005); G. Cognola, E. Elizalde, S. D. Nojiri and S. D. Odintsov and S. Zerbini, Phys. Rev. D73 084007 (2006); G. Calcagni, S. Tsujikawa and M. Sami, Class. Quant. Grav. 223977 (2005); M. 
Sami, A. Toporensky, P. V. Tretjakov and S. Tsujikawa, Phys. Lett. B619193 (2005); S. D. Nojiri and S. D. Odintsov, Gen. Rel. Grav. 361765 (2004); B. C. Paul and M. Sami, Phys. Rev. D70 027301 (2004).

[17] A. K. Sanyal, Phys. Lett. B645 1 (2007).

[18] E. Elizalde, A. N. Makarenko, V. V. Obukhov, K. E. Osetrin, A. E. Filippov, Phys. Lett. B 6441 (2007).

[19] K. Bamba et al, Prog. Theor. Phys. 118879 (2007), arXiv:0707.4334.

[20] N. Dadhich, R. Maartens, P. Papadopoulos and H. Rezina,, Phys. Lett. B4871 (2000).

[21] H. Maeda and N. Dadhich, Phys. Rev. D74 021501 2006, Phys. RevD75 044007 (2007).

[22] A. Molina and N. Dadhich N., arXiv:0804.1194.

[23] B. C. Paul and S. Mukherjee,, Phys. Rev. D422595 (1990).

[24] S. Mukherjee, B. C. Paul, N. K. Dadhich, S. D. Maharaj and A. Beesham,, Emergent Universe in Starobinsky model, gr-qc/0505103.

[25] S. Mukherjee, B. C. Paul, N. K. Dadhich, S. D. Maharaj and A. Beesham, Class. Quantum Grav. 23, 6927 (2006).

[26] E. R. Harrison, Mont. Not. R. Aston. Soc.137 69 (1967).

[27] G. F. R. Ellis and R. Maartens, Class. Quant. Grav. 21223 (2004).

[28] G. F. R. Ellis, J. Murugan and C. G. Tsagas,, Class. Quant. Grav. 21233 (2004); gr-qc/0307112.

[29] D. J. Mulryne, R. Tavakol, J. E. Lidsey and G. F. R. Ellis,, Phys. Rev. D71123512 2005.

[30] S. del Campo, R. Herrera, P. Labrana, JCAP 0711, 030 (2007).

[31] A. Banerjee, T. Bandyopadhyay, S. Chakraborty, Grav. Cosmo. 13, 290 (2007).

[32] A. Banerjee, T. Bandyopadhyay, S. Chakraborty, Gen. Rel. Grav. 40,1603 (2008).

[33] U. Debnath, Class. Quant. Grav. 25205019 (2008).

[34] A. Beesham, S. V. Chervon, S. D. Maharaj, Class.Quant.Grav. 26075017 (2009) 
[35] R. R. Caldwell, Phys. Lett. B 54523 (2002); S. Nojiri and S. D. Odintsov, Phys. Lett. B 562147 (2003); P. Singh, M.Sami, N. Dadhich, Phys. Rev. D 68023522 (2003); L.P. Chimento, R. Lazkoz, Phys. Rev. Lett. 91211301 (2003); B. M. Leith, I. P. Neupane, JCAP 0705019 (2007); V. Faraoni, Class. Quant. Grav. 223235 (2005), B. C. Paul and D. Paul, Pramana, Journal of Physics 711247 (2008).

[36] D. J. Gross and J. H. Sloan, Nucl. Phys. B291 41 (1987); R. R. Metsaev and A. A. Tseytlin, Nucl. Phys. B2933851987.

[37] L. Parker and J. Z. Simon, Phys. Rev. D 47, 1339 (1993), arXiv : gr-qc/9211002,

[38] G. W. Gibbons, Nucl. Phys. B 292, 784 (2003); J. D. Barrow, G. F. R. Ellis, R. Maartens and C. G. Tsagas Class. Quant. Grav. 20 L155 (2003).

[39] G. Boehmer, Lukas Hollenstein, Fracisco S. N. Lobo, Phys. Rev. D 76084005 (2007), gr-qc/0706.1663.

[40] Rituparno Goswami, Naureen Goheer, Peter K. S. Dunsby, Phys. Rev. D 78044011 (2008), arXiv: gr-qc/0804.3528.

[41] Sanjeev S. Seahra, Christian G. Boehmer, Phys. Rev. D 79 064009 (2009), arXiv: gr-qc/0901.0892.

[42] J. D. Barrow, G. F. R. Ellis, R. Maartens and C. G. Tasgas Class. Quant. Grav. 20 L155 (2003); B. Losic and W. G. Unruh Phys. Rev. D 71044011 (2005). 